\title{
Combined aesthetic interventions for prevention of facial ageing, and restoration and beautification of face and body
}

This article was published in the following Dove Press journal:

Clinical, Cosmetic and Investigational Dermatology

30 October 2017

Number of times this article has been viewed

\author{
Sabrina Fabi' \\ Tatjana Pavicic ${ }^{2}$ \\ André $\mathrm{Braz}^{3}$ \\ Jeremy B Green ${ }^{4,5}$ \\ Kyle Seo ${ }^{6}$ \\ Jani AJ van Loghem ${ }^{7}$
}

'Cosmetic Laser Dermatology, San Diego, CA, USA; ${ }^{2}$ Private Practice for Dermatology and Aesthetics, Munich, Germany; ${ }^{3}$ Division of the Policlínica Geral do Rio de Janeiro (PGRJ), Rio de Janeiro, Brazil; ${ }^{4}$ Skin Associates of South Florida, Miami, ${ }^{5}$ University of Miami Department of Dermatology, Miami, FL, USA; 'Seoul National University, Seoul, South Korea; ${ }^{7}$ Doctors Inc., Amsterdam, The Netherlands
Correspondence: Sabrina Fabi

Cosmetic Laser Dermatology, 9339

Genesee Avenue, Suite 300, San Diego, CA 92I 21 , USA

Email sgfabi@gmail.com
Abstract: The Merz Institute of Advanced Aesthetics Expert Summit was held in Prague, Czech Republic, from 19-20 November 2016. The meeting had a distinct advisory board character and invited aesthetic practitioners from all over the world to hear an international faculty present a range of keynote lectures and conduct live injection sessions with an emphasis on recent developments in combination aesthetic interventions for face and body rejuvenation and beautification. Aging is associated with changes in bones, muscles, ligaments, adipose tissue, and skin and, moreover, involves interactions among these tissue types. To achieve the most natural and harmonious rejuvenation of the face, all changes that result from the aging process should be corrected, which generally involves treatment with more than a single agent or technology. Presentations described innovative treatment algorithms for the face and body and focused on patients' desires for natural-looking rejuvenation and how this requires a three-dimensional approach combining products that relax the musculature, volumize, and re-drape the skin. Besides treating the aging face, these procedures are increasingly used to enhance facial features as well as to delay facial aging in younger patients. The presentations covered patients from different ethnicities as well as the treatment of non-facial areas, with a particular focus on the use of Ultherapy ${ }^{\circledR}$ for skin lifting and tightening, and new aesthetic procedures such as Cellfina ${ }^{\circledR}$ and diluted Radiesse ${ }^{\circledR}$. The current report provides a summary of key presentations from the meeting.

Keywords: calcium hydroxylapatite, Cellfina, hyaluronic acid, incobotulinumtoxinA, Ultherapy

\section{Introduction}

More than 400 experts in dermatology, plastic surgery, and aesthetic medicine from 60 countries gathered in Prague to participate in the Merz Expert Summit on combined aesthetic interventions for the face and body. This was an interactive event with a bidirectional transfer of knowledge combining many medical specialties. To stimulate participation from this broad international audience, simultaneous translations from English into six languages (French, German, Italian, Portuguese, Russian, and Spanish) were provided.

The aim of the meeting was to inform physicians about recent advances in aesthetic medicine with a focus on combined interventions, and to gain audience feedback about their experience and usual practices. Over the course of 2 days, 20 sessions took place covering a range of topics including: combining aesthetic interventions for the prevention and rejuvenation of aged skin, multicultural aspects in the perception of beauty and patient expectations, treatment options for non-facial areas, and emerging 
procedures for body beautification and rejuvenation such as Cellfina ${ }^{\circledR}$, Ultherapy $^{\circledR}$, and diluted Radiesse ${ }^{\circledR}$ (calcium hydroxylapatite, CaHA) (all three from Merz North America, Inc., Raleigh, NC, USA).

Combined aesthetic interventions have widespread application in clinical practice, but results are infrequently reported at scientific meetings and in the medical literature, as the area is difficult to study; existing reports are mainly anecdotal. Many of the speakers reminded the audience to look beyond the face and consider the neck, décolletage, and the hands. Body rejuvenation procedures for the buttocks, thighs, arms, and abdomen were also reviewed.

This report summarizes presentations from the meeting on recent developments in combined aesthetic interventions, including which treatments to undertake first and which to combine to provide patients with the best possible outcomes.

\section{Rationale for combined aesthetic interventions}

For the most natural and harmonious rejuvenation of the face, all changes that result from the aging process should be corrected. Dr Tatjana Pavicic (Private Practice, Munich, Germany) reminded the audience that aging is a result of the interplay of changes occurring in all five facial anatomical layers: skeleton, ligaments, muscles, adipose tissue, and skin. To target these, multilayer, combined intervention is required to relax, volumize, resurface, and re-drape facial skin.

No single technology, filler, or neuromodulator can achieve all the results desired in treating the aging face. The question is when and how to combine different aesthetic interventions safely and effectively for the face, hands, neck, and décolletage.

\section{Tips and techniques for combined aesthetic interventions}

Dr Sabrina Fabi (Cosmetic Laser Dermatology, San Diego, CA, USA) described how combined aesthetic interventions are more than just the sum of the components. Optimal outcomes are dependent upon choosing the appropriate tool and ensuring that it is used correctly. A deep understanding of product characteristics, anatomy, and the physiology of aging is essential to know when, where, and how to use different modalities to provide facial harmony.

Live injection sessions throughout the 2-day meeting illustrated some of the injection techniques that can be used when combining aesthetic interventions, with a strong emphasis on safety. Physician experience is essential for satisfied patients and, as pointed out by Dr Jani van Loghem
(Doctors Inc., Amsterdam, The Netherlands), ongoing training is essential for all levels of professionals to ensure safety and avoid complications as well as achieve the best outcomes for patients. ${ }^{1}$

Drs Fabi and Kate Goldie (European Medical Aesthetics Ltd, London, UK) reviewed recently published consensus recommendations for combined aesthetic interventions, ${ }^{2,3}$ which are the first guidelines published to date to provide specific recommendations for the optimal combination, treatment timing, and sequence with botulinum toxin (eg, incobotulinumtoxinA; Xeomin ${ }^{\circledR} /$ Bocouture $^{\circledR}$; Merz Pharmaceuticals GmbH, Frankfurt am Main, Germany), calcium hydroxylapatite (CaHA; Radiesse ${ }^{\circledR}$ ), and hyaluronic acid (HA) fillers (eg, Cohesive Polydensified Matrix [CPM] HA fillers; Belotero ${ }^{\circledR}$; Anteis S.A, Geneva, Switzerland), and microfocused ultrasound with visualization (Ultherapy ${ }^{\circledR}$ ) for early intervention and aesthetic restoration of the face as well as other areas of the body.

In terms of the sequence of multiple treatments in one area, there was strong consensus to consider spacing individual treatments by $1-2$ weeks to allow resolution of local side effects and/or to assess the results; however, if undertaken on the same day, incobotulinumtoxinA, CPM-HA, and/or CaHA may be performed in any sequence. For combination therapy with multiple agents on the same day, Ultherapy ${ }^{\circledR}$ should be performed before the filler or incobotulinumtoxinA. ${ }^{2}$

The consensus presents the order and sequence for early intervention and restoration of the upper face, midface, and lower face. ${ }^{2}$ In the upper face, incobotulinumtoxinA is recommended for muscle control; for patients presenting with volume depletion of the forehead and temples, the recommendation is to start with an HA. In the midface, CPM-HA and/or CaHA are recommended for volume loss in the cheek without laxity; however, in the presence of laxity, the consensus recommends starting with Ultherapy ${ }^{\circledR}$ followed by CPM-HA and/or CaHA. In the lower face, combination treatment using incobotulinumtoxinA to relax any areas being pulled down, and CPM-HA and/or CaHA to create structure and volume, is recommended. For the neck, incobotulinumtoxinA is recommended as first-line treatment, followed by Ultherapy ${ }^{\circledR}$ and low-viscosity CPM-HA and/or diluted CaHA as second- and third-line treatments, respectively, to stimulate neocollagenesis and improve skin texture. ${ }^{3}$ For the décolletage, low-viscosity CPM-HA and/or diluted CaHA to stimulate neocollagenesis are recommended for early intervention. However, in cases of true restoration, Ultherapy ${ }^{\circledR}$ is recommended as first-line treatment followed by lowviscosity CPM-HA and/or diluted CaHA on the same day. 


\section{Combined measures for prevention of facial skin aging}

Facial skin aging is caused by intrinsic and extrinsic mechanisms. Intrinsic skin aging represents the normal course of aging for all tissues, whereas extrinsic aging is mainly caused by exposure to UV radiation, pollution, and cigarette smoking that is superimposed on intrinsic skin aging. Exposed areas of the body such as the face are, therefore, subjected to both types of skin aging. Dr André Braz (Policlínica Geral do Rio de Janeiro, Rio de Janeiro, Brazil) presented his case studies, demonstrating treatment recommendations for prevention in individuals with no or minimal signs of aging from a consensus meeting involving 14 specialists in aesthetic medicine that took place in April 2016 in Monaco. ${ }^{4}$ Skin glow and skin texture have a significant impact on attractiveness. Healthy skin can be retained with the use of topical skincare products, including daily UV protection, moisturizers, and topical antioxidants. These will also prevent further damage in patients presenting with environmentally induced irregular pigmentation. For the prevention or early treatment of superficial skin laxity and/or fine wrinkles, options would include topical skincare (eg, UV protection, antioxidants, retinoids, moisturizers, DNA repair enzymes), smoking abstinence or cessation, and some device-based rejuvenating procedures (eg, intense pulsed light, radiofrequency).

For patients presenting with early static lines and folds ( $\leq$ grade 2 on contraction and $\leq$ grade 1 at rest on assessment scales), the consensus recommended preventative injections with incobotulinumtoxinA. Soft-tissue fillers are recommended for patients with laxity of deep fibrous structures to provide support. Ultherapy ${ }^{\circledR}$ and radiofrequency may also be used in areas demonstrating laxity. Early intervention with fillers is recommended in individuals with facial volume loss that is asymmetric or in patients without strong bony support.

\section{Combined measures for beautification: different ethnicities, different approaches}

To date, most studies and published recommendations on the use of aesthetic interventions (especially their use in combination) are based on Caucasian populations. However, individuals from different ethnic backgrounds vary in both facial appearance and baseline structural facial anatomy. These factors must be taken into account when planning a treatment approach. ${ }^{5,6}$

Dr Kyle Seo (Seoul National University Hospital, Seoul, South Korea) presented his perspective on ethnic differences, with a focus on East Asian patients. The Asian face is typically wider and flatter compared with the narrower Caucasian face. The use of botulinum toxin to counteract masseter hypertrophy, temporalis hypertrophy, and parotid gland enlargement - all of which contribute to the wide face that is characteristic of Asian patients - is a popular indication to make the face slimmer and appear more oval. Asian patients typically require lower doses of toxin than Caucasian patients (8-12 U for glabella and 3-6 U for horizontal forehead lines), probably because their facial muscles are less active and, therefore, develop fewer mimetic lines - a characteristic that could be race- or culture-related. In addition, Asian people have a shorter corrugator muscle and thicker skin than Caucasian people. A further popular indication for botulinum toxin in Asia is for body reshaping, particularly slimming of the calf, deltoid, trapezius, and quadriceps femoris muscles to create a smoother, slimmer, and elongated body contour.

The use of fillers to add volume to the vertical midface, including the forehead, nose, sunken cheeks, and chin is also widely indicated in Asians to produce a slimmer and more three-dimensional face, and is useful not only for facial rejuvenation, but also for beautification through the enhancement of facial contours. In contrast, horizontal midface augmentation such as accentuating the cheek bones, popular in Caucasian patients, would only make an Asian face look wider and should be avoided. It should be noted that the nose is a challenging part of the face to inject, even in experienced hands, because of the risk of accidental intravascular injection, particularly in individuals whose vascular anatomy has altered (eg, after rhinoplasty).

The focus of Dr Braz was on Latin American beauty, where the main features are a well-defined lower face with a clear jawline, a wide midface, and a feminine body. He illustrated how the CPM-HA range can be used to achieve this facial beauty concept in a global face approach through treatment of volume depletion and modeling of facial contours. Target areas included the cheek, tear trough, temporal hollows, chin, and nasal dorsum. With the use of injection videos, cadaver dissections, and skulls, he illustrated the precise anatomy of each injection area, and how this defines the correct layer of injection, and selected injection techniques to efficiently and safely augment the different regions of the face.

Merz is currently developing a series of scales, specifically for guiding aesthetic interventions and assessing treatment outcomes in Asian patients. Examples are the facial shape scales, which categorize faces as predominantly oval, oblong, round, or square, and the calf scales, which are intended to provide guidance for calf-slimming procedures. 


\section{Ultherapy ${ }^{\circledR}$ - face and beyond}

Ultherapy ${ }^{\circledR}$ was developed to meet the growing public demand for noninvasive skin lifting and tightening procedures. The device combines microfocused ultrasound (MFU) with high-resolution ultrasound imaging to allow the user to visualize where the MFU energy will be applied. Use of ultrasound visualization to target the correct tissues is essential as there are patient variations in skin thickness and anatomical features (on the basis of gender, age, body weight, ethnicity, etc). Visualization is the only way to select the appropriate treatment depth and transducer. Dr Fabi recommended the use of two transducer depths for synergistic effects, but highlighted the importance of ensuring that both depths are appropriate using visualization.

Furthermore, recommendations were provided to help avoid adverse events related to the device. The most commonly reported adverse event associated with MFU-V is brief discomfort during the treatment session. A combination of topical anesthesia and oral analgesics (eg, $2 \times 400 \mathrm{mg}$ ibuprofen) are routinely used prior to treatment to reduce pain levels. The quality of coupling/contact and the amount of ultrasound gel used can affect whether energy is being efficiently delivered at the appropriate depth. Excess ultrasound gel can result in welts on the skin surface due to energy delivery at superficial depths (Figure 1). Dermal papules can arise following pulse stacking (ie, repeated delivery of energy to the same location). Visualization combined with a detailed knowledge of local anatomy should be used to avoid delivering energy to facial nerves, which can result in neuropraxia.

Ultherapy ${ }^{\circledR}$ has been studied for skin tightening/lifting in multiple areas, including the décolletage, ${ }^{7,8}$ buttocks, ${ }^{9}$ knees, ${ }^{10,11}$ medial thighs, ${ }^{10}$ arms/elbows, ${ }^{10,12}$ and the abdomen. ${ }^{13}$ Figure 2 illustrates the use of this technique for skin tightening on the buttocks. Dr Fabi emphasized that achieving the patient's aesthetic goals may require the combination of Ultherapy ${ }^{\circledR}$ with other aesthetic treatments (eg, filler and toxin injections, liposuction, cryolipolysis). The use of Ultherapy ${ }^{\circledR}$ to treat skin laxity on the body is an area that is still being actively researched. Dr Fabi is investigating Ultherapy ${ }^{\circledR}$ for the treatment of abdominal laxity in a study that aims to enroll 24 subjects to receive two treatments 90 days apart (700 lines per abdomen at both the 3 and $1.5 \mathrm{~mm}$ depths).

\section{Emerging body beautification and rejuvenation procedures}

Cellulite - the Cellfina ${ }^{\circledR}$ system

Cellulite affects the vast majority of women, even those who are fit and slim, but a better understanding of its pathophysiology

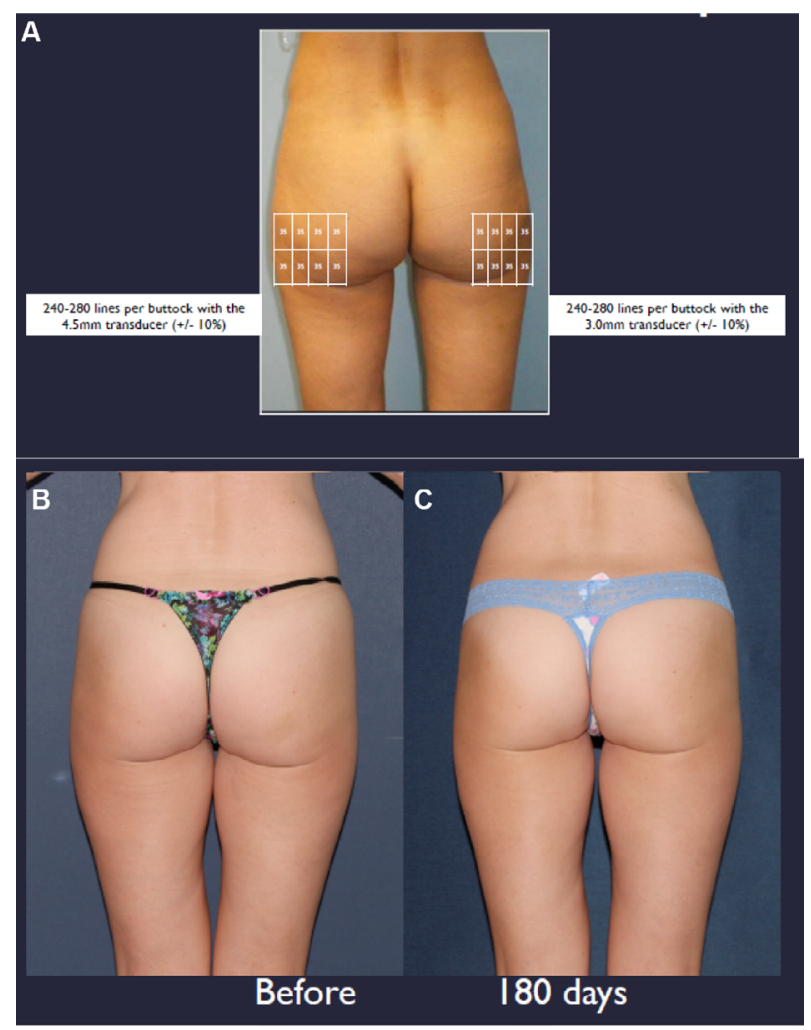

Figure 2 (A) Ultherapy ${ }^{\circledR}$ buttock treatment map. (B) Before treatment. (C) 180 days post-treatment (Courtesy of Dr Sabrina Fabi).

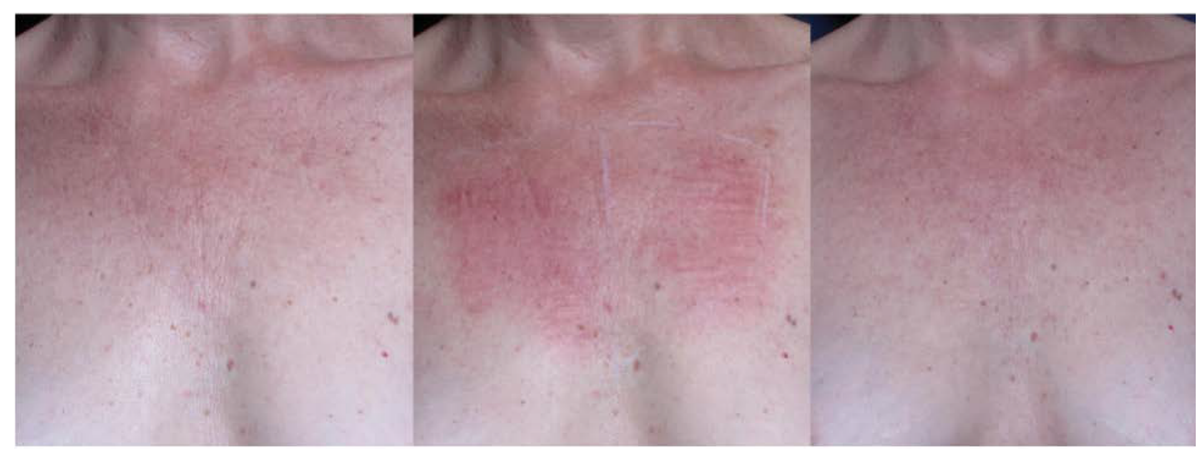

Figure I Transient welts following Ultherapy ${ }^{\circledR}$ as a result of application of excess gel and their resolution 3 weeks later (Courtesy of Dr Sabrina Fabi). 
has led to the development of the only long-term treatment cleared by the US FDA for cellulite treatment. Dr Jeremy Green (Skin Associates of South Florida, Miami, and University of Miami Department of Dermatology, FL, USA) introduced the Cellfina $^{\circledR}$ system (Merz North America, Inc.), which has been engineered to treat cellulite by addressing the underlying physical structures that cause the appearance of dimples. Imaging and microscopy analyses have shown that fibrous septae oriented perpendicular to the skin surface connect the dermis with deeper fascial layers. Cellulite on the thighs and buttocks arises when these septae pull the undersurface of the skin causing dimpling with protrusion of the superficial fat compartments. This results in a tufted or mattress-like appearance of the skin. Cellfina ${ }^{\circledR}$ targets the release of fibrous septae that cause the appearance of dimples. The vacuum-assisted tissue stabilization platform enables minimally invasive subcision of the fibrous septae to be undertaken at one of two controlled depths (6 or $10 \mathrm{~mm}$ ). The precise targeting and depth control afforded by the Cellfina ${ }^{\circledR}$ system distinguishes it from manual subcision, which is associated with greater posttreatment morbidity. Figure 3 illustrates the results of Cellfina ${ }^{\circledR}$ at 5 months posttreatment.

Dr Green highlighted the results of the Cellfina ${ }^{\circledR}$ pivotal trial that led to the US FDA clearance of the device for longterm cellulite treatment. The pivotal study enrolled 55 subjects who were followed for 3 years after a single treatment. ${ }^{14}$ On a 5-point cellulite severity scale, the mean improvement was 2 points at 1,2, and 3 years after the initial procedure. The proportion of subjects with at least a 1-point improvement in cellulite severity remained at $91 \%$ after 3 years, and subject satisfaction was $\geq 93 \%$ at 1,2 , and 3 years posttreatment. No serious adverse events were observed, with most subjects reporting only expected treatment-related effects (eg, bruising, induration).

In addition, Dr Green summarized findings from a realworld registry study on patients receiving Cellfina ${ }^{\circledR}$ treatment (CRUISE). The study has enrolled 52 patients and collected data on the procedure itself (eg, time to complete procedure),

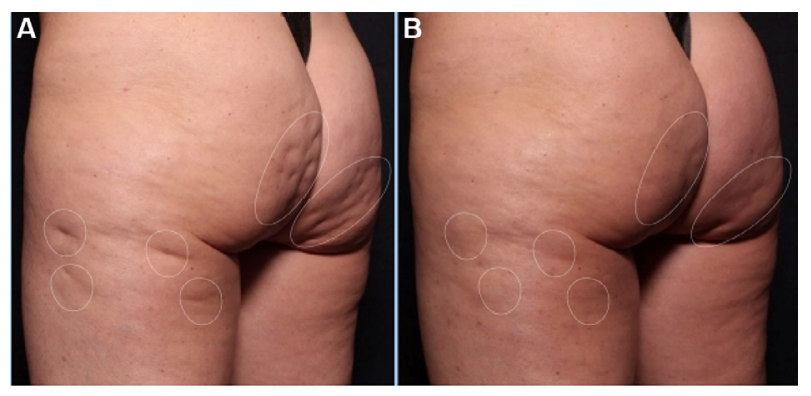

Figure 3 (A) Before and (B) after photos of Cellfina ${ }^{\circledR}$ treatment in a 50-year old woman (Courtesy of Dr Jeremy B Green). patient-reported pain scores, adverse events, and quality of life, and found that the successful results of the pivotal trial did, in fact, translate into clinical practice.

\section{Diluted calcium hydroxylapatite}

The use of diluted CaHA for skin tightening is a novel technique that takes advantage of the biostimulatory properties of CaHA mediated by its calcium hydroxylapatite microspheres, which have been shown in different histological studies to stimulate neocollagenesis, neoelastogenesis, angiogenesis, and fibroblast proliferation. ${ }^{15,16}$ This makes it an effective treatment for improving contours and firmness in individuals with age-associated upper arm skin changes.

Drs van Loghem and Valeria Cogorno Wasylkowski (Clinic Novosalud, Madrid, Spain) illustrated a skintightening procedure in a live demonstration of upper arm and elbow rejuvenation in a female patient. The solution mix comprised $3 \mathrm{~mL}$ CaHA, $8 \mathrm{~mL}$ saline, and $1 \mathrm{~mL}$ lidocaine solution. Dr van Loghem demonstrated two different techniques. In the right arm, he used four entry points in the upper and lower peripheries of the anterior brachial zone and injected diluted CaHA subdermally with a $5 \mathrm{~cm}$-long $25 \mathrm{G}$ cannula using a crosshatching technique with retrograde linear threads (Figure 4A). In the left arm, he illustrated a vertical retrograde linear threading technique with a $28 \mathrm{G}$ needle using multiple entry points (Figure 4B). This approach is faster than with the cannula, but may cause more bruising. Photos before and after treatment with CaHA for the upper arm are shown in Figure 4C and D. Dr Cogorno Wasylkowski used a $25 \mathrm{G}$ needle and demonstrated the vectoring technique
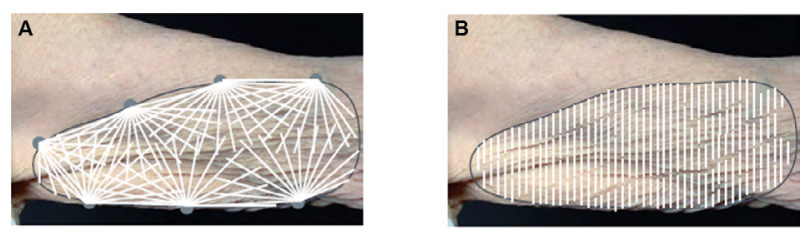

C D

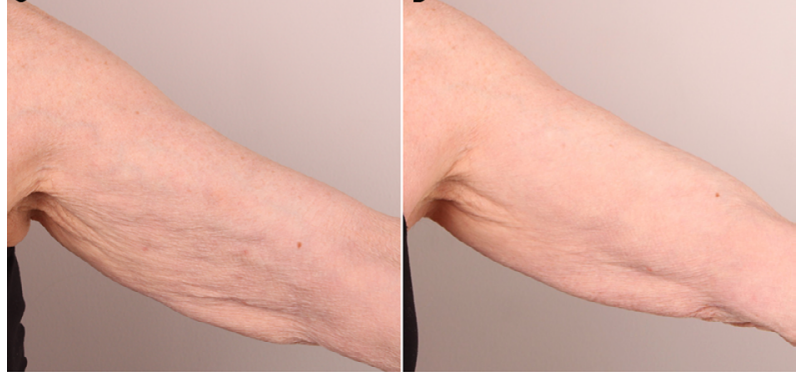

Figure 4 Upper arm injection techniques with diluted calcium hydroxylapatite for skin tightening. (A) Crosshatching technique with retrograde linear-threads performed with a $5 \mathrm{~cm}$-long $25 \mathrm{G}$ cannula. (B) Vertical retrograde linear threading technique performed with a $28 \mathrm{G}$ needle. (C) Before and (D) after treatment with $\mathrm{CaHA}$ for the upper arms (Courtesy of Dr Jani van Loghem). 
for treatment of the right posterior arm, as described previously in detail. ${ }^{17}$ The approach has achieved good results with a duration of approximately 1 year, depending on the degree of skin laxity. Optimal results can be expected at 3-4 months.

Diluted CaHA has also been effectively used in the neck and décolletage to improve skin mechanical properties and stimulate neocollagenesis - a procedure pioneered by Prof Yana Yutskovskaya (Pacific State Medical University, Vladivostock, Russia). The procedure triggers a two-step process of neocollagenesis, in which collagen type I gradually replaces collagen type III, ${ }^{16}$ and elastogenesis. The technique employed by ProfYutskovskaya uses multiple $(0.025 \mathrm{~mL}$ per pass), retrograde linear threading ( $0.5 \mathrm{~cm}$ apart) of $1: 2-1: 6$ diluted CaHA (diluted with saline solution, plus lidocaine for pain) placed in the subdermal plane with a needle or cannula, followed by massage with gel or cream. With this procedure, bruising is virtually unseen. Prof Yutskovskaya stated that she has carried out this mesotherapy technique combined with Ultherapy ${ }^{\circledR}$ for more than 2 years, and it is now the top procedure at her clinic.

\section{Hand rejuvenation with calcium hydroxylapatite ${ }^{\circledR}$}

Hands: exploring injectable treatment options and techniques

In addition to the face, neck, and décolletage, it is also important not to forget other exposed areas of the body such as the hands. Hands can give away a person's age as easily as the face. ${ }^{18}$

CaHA is the first and currently only dermal filler approved by the US FDA to correct volume loss in the hands. In a recent multicenter, randomized, controlled, single-blinded study, treatment has been shown to have a duration of effect of at least 12 months following a single procedure and is very well tolerated, with adverse events restricted to mild-tomoderate injection-site reactions and without issues relating to hand function. ${ }^{19}$

The correct depth of injection when treating the hand is in the subdermal plane and not in the tendinous layer. One technique to achieve this was explained and demonstrated by Dr Pavicic. The Skin Scraping Threading Technique (SSTT $\left.{ }^{\circledR}\right)$ for hand augmentation places filler in the undersurface of the dermis (the dorsal superficial lamina). ${ }^{20}$ The SSTT $^{\circledR}$ was developed to allow safe and accurate injection of filler by "scraping at the bottom of the dermis" with a cannula. Using this technique, filler placement is restricted to the fascial layer and should be performed with a 7-cm long, non-flexible cannula (22G $23 / 4$ ") and a proximal insertion point. The filler is injected with the cannula bevel facing upwards.

\section{Conclusion}

With our increased understanding of facial anatomy and new and refined uses for different aesthetic tools and products, the spectrum of facial aesthetics is constantly evolving. A clear message from this expert summit was that, to offer patients the best possible outcomes, we should no longer think about individual treatments but look at the whole face and at different tissue depths to determine the appropriate treatment approach. Recognizing that more than one procedure will be required to correct all the issues, injectables and other modalities such as topical skincare and energy-based therapies can be combined for optimal results. In addition to rejuvenation, physicians also have the right tools in their hands to prevent or delay skin aging, which can be offered to the increasing numbers of younger patients visiting aesthetic practices.

The meeting also highlighted that an understanding of different global concepts of beauty is essential for achieving optimal outcomes. In each ethnic group, the best outcomes are obtained by maximizing a patient's good structural characteristics and improving structural deficiencies common to that group.

The focus of the meeting was not only the face, with many of the speakers reminding the audience to consider other areas that can give away a person's age including the neck, décolletage, and the hands. Furthermore, with emerging procedures such as Cellfina ${ }^{\circledR}$ for cellulite, and Ultherapy ${ }^{\circledR}$ and diluted $\mathrm{CaHA}$ for skin tightening and improving skin mechanical properties, the Merz Aesthetics portfolio has been broadened to provide innovative approaches for body rejuvenation and beautification.

\section{Acknowledgments}

The authors thank all speakers for contributing to the content of the Expert Summit, and Jenny Grice for editorial assistance, which was funded by Merz Pharmaceuticals $\mathrm{GmbH}$, Frankfurt am Main, Germany.

Merz Pharmaceuticals GmbH, Frankfurt am Main, Germany, supported the Expert Summit and the writing of these proceedings. The content of the publication reflects the experts' independent opinions and experiences. Merz Pharmaceuticals $\mathrm{GmbH}$ does not encourage or endorse the use of any product manufactured or distributed by the company in ways that are not approved by applicable notified bodies or regulatory agencies.

\section{Disclosure}

The authors have disclosed financial relationships with the following companies: 
S Fabi has conducted research for Allergan, Galderma, Merz, Alastin, Colorscience, Valeant, and Revance, is a speaker for Allergan, Galderma, Merz, and Valeant, and a consultant/advisory board member for Allergan, Galderma, Merz, and Valeant.

T Pavicic has received honoraria from Merz Aesthetics and Galderma Pharma SA for speaker activities. She is a consultant for Merz Aesthetics and Galderma Pharma SA and serves on advisory boards for these companies.

A Braz is a consultant and speaker for Allergan, Galderma, Merz, and Palomar.

JB Green is an advisory board member, research investigator and speaker for Allergan, Galderma, and Merz, an investigator and advisory board member for Sienna and Lutronic, an investigator for Brickell Biotech, Cutera, Valeant, Teoxane, Revance, and Neothetics, and holds stock in Illustris.

$\mathrm{K}$ Seo serves as a clinical investigator and/or consultant for Allergan, Merz Pharmaceuticals, Q-Med/Galderma, Medytox, LG Life Sciences, and Daewoong.

JAJ van Loghem is a consultant for Merz Aesthetics.

\section{References}

1. van Loghem JA, Humzah D, Kerscher M. Cannula versus sharp needle for placement of soft tissue fillers: an observational cadaver study. Aesthet Surg J. Epub 2016 Dec 16.

2. Carruthers J, Burgess C, Day D, et al. Consensus recommendations for combined aesthetic interventions in the face using botulinum toxin, fillers, and energy-based devices. Dermatol Surg. 2016;42(5):586-597.

3. Fabi SG, Burgess C, Carruthers A, et al. Consensus recommendations for combined aesthetic interventions using botulinum toxin, fillers, and microfocused ultrasound in the neck, décolletage, hands, and other areas of the body. Dermatol Surg. 2016;42(10):1199-1208.

4. Landau M, Anand CV, Besins T, et al. First consensus on primary prevention and early intervention in aesthetic medicine. J Drugs Dermatol. 2017;16(9):846-854.

5. Rho NK, Chang YY, Chao YY, et al. Consensus recommendations for optimal augmentation of the Asian face with hyaluronic acid and calcium hydroxylapatite fillers. Plast Reconstr Surg. 2015;136(5):940-956.

6. Sundaram H, Huang PH, Hsu NJ, et al; Pan-Asian Aesthetics Toxin Consensus Group. Aesthetic applications of botulinum toxin A in Asians: an international, multidisciplinary, Pan-Asian consensus. Plast Reconstr Surg Glob Open. 2016;4(12):e872.
7. Fabi SG, Massaki A, Eimpunth S, Pogoda J, Goldman MP. Evaluation of microfocused ultrasound with visualization for lifting, tightening, and wrinkle reduction of the décolletage. JAm Acad Dermatol. 2013;69(6): 965-971.

8. Fabi SG, Goldman MP, Dayan SH, Gold MH, Kilmer SL, Hornfeldt CS. A prospective multicenter pilot study of the safety and efficacy of microfocused ultrasound with visualization for improving lines and wrinkles of the décolleté. Dermatol Surg. 2015;41(3):327-335.

9. Goldberg DJ, Hornfeldt CS. Safety and efficacy of microfocused ultrasound to lift, tighten, and smooth the buttocks. Dermatol Surg. 2014;40(10):1113-1117.

10. Alster TS, Tanzi EL. Noninvasive lifting of arm, thigh, and knee skin with transcutaneous intense focused ultrasound. Dermatol Surg. 2012;38(5):754-759.

11. Gold MH, Sensing W, Biron J. Use of micro-focused ultrasound with visualization to lift and tighten lax knee skin (1.). J Cosmet Laser Ther. 2014;16(5):225-229.

12. Rokhsar C, Schnebelen W, West A, Hornfeldt C. Safety and efficacy of microfocused ultrasound in tightening of lax elbow skin. Dermatol Surg. 2015;41(7):821-826.

13. Sasaki HG, Tevez A. Microfocused ultrasound for nonablative skin and subdermal tightening to the periorbitum and body sites: preliminary report on eighty-two patients. J Cosmet Dermatol Sci Appl. 2012;2(2A):108-116.

14. Kaminer MS, Coleman WP 3rd, Weiss RA, Robinson DM, Grossman J. A multicenter pivotal study to evaluate tissue stabilized-guided subcision using the Cellfina device for the treatment of cellulite with 3-year follow-up. Dermatol Surg. Epub 2017 Jun 28.

15. Yutskovskaya YA, Kogan EA. Improved neocollagenesis and skin mechanical properties after injection of diluted calcium hydroxylapatite in the neck and décolletage: a pilot study. J Drugs Dermatol. 2017;16(1):68-74.

16. Yutskovskaya Y, Kogan E, Leshunov E. A randomized, split-face, histomorphologic study comparing a volumetric calcium hydroxylapatite and a hyaluronic acid-based dermal filler. J Drugs Dermatol. 2014;13(9):1047-1052.

17. Cogorno Wasylkowski V. Body vectoring technique with Radiesse(®) for tightening of the abdomen, thighs, and brachial zone. Clin Cosmet Investig Dermatol. 2015;8:267-273.

18. Bains RD, Thorpe H, Southern S. Hand aging: patients' opinions. Plast Reconstr Surg. 2006;117(7):2212-2218.

19. Goldman MP, Moradi A, Gold MH, et al. Calcium hydroxylapatite dermal filler for treatment of dorsal hand volume loss: results from a 12-month, multicenter, randomized, blinded trial. Dermatol Surg. Epub 2017 May 25.

20. Lefebvre-Vilardebo M, Trevidic P, Moradi A, Busso M, Sutton AB, Bucay VW. Hand: clinical anatomy and regional approaches with injectable fillers. Plast Reconstr Surg. 2015;136(5 Suppl):258S-275S.
Clinical, Cosmetic and Investigational Dermatology

\section{Publish your work in this journal}

Clinical, Cosmetic and Investigational Dermatology is an international, peer-reviewed, open access, online journal that focuses on the latest clinical and experimental research in all aspects of skin disease and cosmetic interventions. This journal is included on PubMed. The manuscript management system is completely online

\section{Dovepress}

and includes a very quick and fair peer-review system, which is all easy to use. Visit http://www.dovepress.com/testimonials.php to read real quotes from published authors 